\title{
EVALUATION OF SOME FRUIT CHARACTERISTICS OF JUJUBE (ZIZIPHUS JUJUBA MILL) GENOTYPES IN MANISA, TURKEY
}

\author{
ACARSOY BILGIN, N. \\ Department of Horticulture, Faculty of Agriculture, Ege University, Izmir, Turkey \\ (Orcid ID: 0000-0002-5018-6347; e-mail: nihalacarsoy@yahoo.com) \\ (Received $4^{\text {th }}$ Oct 2019; accepted $8^{\text {th }}$ Jan 2020)
}

\begin{abstract}
Jujube fruits are widely consumed all over the world as food and herbal medicine due to their health benefits. It is known as a species with a wide variety of products. It can be found naturally throughout the world, including Turkey. Jujube is particularly popular nowadays for its high antioxidant activity and phenolic content. In this study conducted on 10 genotypes selected in Manisa/Demirci (Turkey) pomological, biochemical and organoleptic properties of fruit genotypes were investigated. The results stated, the characteristics examined differed according to genotypes. The highest TSS, phenol and antioxidant content were $45.34 \%, 322.39 \mathrm{mg} \mathrm{GAE} / \mathrm{g}$ fw and $96.75 \mu \mathrm{mol} \mathrm{TE} / \mathrm{g}$ fw, respectively. Some genotypes are thought to be beneficial for public health as well as for table use due to their phytochemical contents.
\end{abstract}

Keywords: selection, pomological, biochemical, organoleptic, statistical

\section{Introduction}

Jujube (Ziziphus jujuba Mill) is one of the temperate fruit species. This plant is resistant to extreme rain fall and is not affected by drought can survive at heights up to $1700 \mathrm{~m}$ above sea level (Ecevit et al., 2002). Jujube is widely grown in almost all over the world (Mukhtar et al., 2004; Gozlekci et al., 2015). In China, it has been cultivated in for many years. It is naturally found in Russia, India, Middle East, Anatolia, Southern Europe and North Africa. There are 6 genus including Colletia, Frangula, Hovenia, Paliurus, Rhamnus, Ziziphus and 25 species in the native flora of Turkey (Anşin and Özkan, 1997). Among them, Ziziphus jujuba and Ziziphus mauritiana are grown for fruits (İslam et al., 2006).

Jujube is usually growing home gardens and as a border tree in a field edge. It grows in Marmara, Western and Southern Anatolia in Turkey (Yücel, 2005). In this respect, Isparta, Hatay, Iskenderun, Antalya, Kayseri, Bursa, Çanakkale are important production centers (Karıncal1, 2003). Also besides, Çoruh Valley, Manisa/Demirci and Denizli/Çivril noteworthy. In this area, there is abundant natural flora and different species are observed (Yaşa, 2016).

In recent years, fruit species with high antioxidant activity, phenolic and vitamin contents are of great interest (Wojdylo et al., 2016). Thereby, jujube has a significant impact on human nutrition all over the world. Fruits are delicious and eaten as fresh, dried, candy, jam, juice, wine, syrup, tea bag sand compotes (Liu, 2006).

The bioactive components show large variations according to genotypes and growing, and ripening (Choi et al., 2011; Koley et al., 2016). Total phenol contents and antioxidant activity of jujube fruits were found to be high in previous study (Kamiloglu et al., 2009). They have significant levels of vitamin A, B, C and content many minerals (Wojdyloa et al., 2016). Accordingly, it is an excellent source of nutrients and phytochemicals and can also contribute to a healthy diet. In this way, it also has a protective feature against various diseases with its composition. Because of these properties, the fruit is well-known as a nourishing food also seeds; roots and leaves are 
known as folk medicine (Mukhtar et al., 2004; Abdel-Zaher et al., 2005; Li et al., 2005). Additionally, fruits contain sugar, tannin and mucilage substances. For this reason, it is recommended that diabetics consume fruit directly. This further increases the importance of the product.

Determining genetic diversity is a prerequisite for conservation of genetic resources, due to the loss of many features of gene erosion. This is important for the sustainability of biodiversity. In this economically valuable species, breeders are researching their genetic resources (Ahmad et al., 2019; Dahlia et al., 2019; Sharif et al., 2019). In the development of new varieties, hybridization breeding takes place over a long period and is difficult. Thanks to the genetic diversity in nature, selection breeding is preferred. Morphological and molecular markers are used to determine genotypes in the population (Ahmad et al., 2019; Chen et al., 2019; Dahlia et al., 2019). As is known, morphological evaluation is easy and inexpensive (Zhang et al., 2015). In this way, breeders can identify elite genotypes by investigating plant, fruit and sensory characteristics (Uddin and Hussain, 2012; Godi and Joshi, 2016).

In Turkey, similarly to many other fruits, this species has a gene pool. The characteristics of jujube genotypes have been determined in different locations in our country but there is no standard variety. This will contribute to breeding programs which new genotypes with different characteristics will be developed. Considering these explanations in the current study, pomological, biochemical and organoleptic properties of 10 genotypes were identified in Manisa/Demirci location.

\section{Materials and methods}

In this study, 10 local jujube genotypes selected from Manisa/Demirci were used as material (Fig. 1). Sampling was performed to represent genetic diversity in the experimental area. In phenotypic observations, individuals with different fruit characteristics such as fruit size, color and taste were selected. For each genotype, 30 fruits were analyzed. Samples were collected during the commercial maturation period (September 2018).

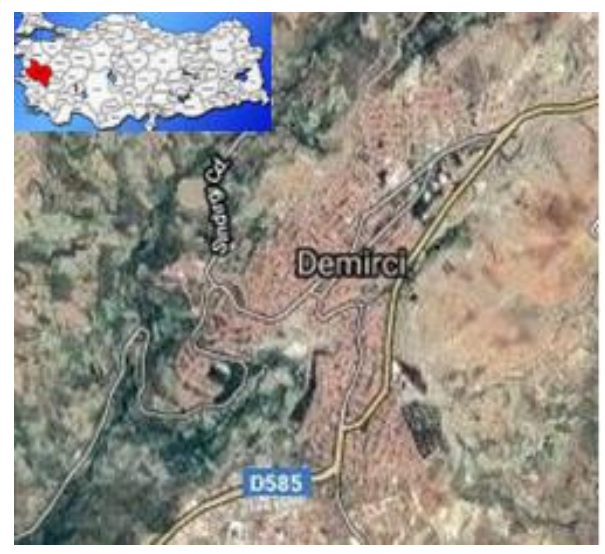

Figure 1. The location of the experimental area

Fruit and stone weights of the harvested samples were determined, and average flesh/stone ratios were calculated. The fruit width and length were measured. Fruit ground colors were stated twice at the equatorial regions of both cheeks for five fruits 
with "CR300 model Minolta Colorimeter". The values of $\mathrm{L}^{*}, \mathrm{a}^{*}, \mathrm{~b}^{*}$ were measured and chrome $\left(\mathrm{C}^{*}\right)$ and hue angle $\left(\mathrm{h}^{\circ}\right)$ were determined based on these values (Karaçall, 2012). TA values (\%) were measured such that $5 \mathrm{ml}$ of fruit juice was completed to 100 $\mathrm{ml}$ with distilled water and $0.1 \mathrm{~N}$ of $\mathrm{NaOH}$ was added to reach a $\mathrm{pH}$ of 8.00-8.10. TSS content in fruit juice was found by a digital refractometer (\%), and the $\mathrm{pH}$ values were read by a $\mathrm{pH}$ meter (Karaçal1, 2012). Total phenol content and antioxidant activity were determined in the fruit samples prepared by Thaipong et al. (2006) method. Total phenol content was determined by the Folin-Ciocalteu method (Swain and Hillis, 1959). The results were expressed as $\mathrm{mg}$ gallic acid equivalent (GAE)/100 $\mathrm{g}$ fresh weight (FW). The FRAP method was used to determine the antioxidant activities. The results were given as Trolox equivalent (TE)/g dm (Benzie and Strain, 1996).

Jujube fruits were evaluated regarding flavor, texture and general acceptability by Meligaared et al. (1991) and Uddin and Hussain (2012). According to this, flavor: no taste (1), elaeagnus-apple (3), apple-pear (5), elaeagnus (7), apple (9); texture: soft (3), medium (5), hard (7) and acceptability: poor (1), fair (3), medium (5), good (7), very good (9) were evaluated with scale ranged from dislike (1) to like (9). At this stage, each genotype was tasted by 10 panelists.

Results were subjected to a variance analysis by using SPSS Statistics 20 statistical package program. Significant differences between averages were defined by Duncan test at the $\mathrm{P}<0.05$ significant level. The mean, minimum, maximum, and standard deviation values of the properties examined were determined. Moreover, the relationship among these values was revealed by conducting Pearson's correlation analysis. Differences or similarities of genotypes were evaluated according to their analyzed properties by applying PCA to the findings obtained. Moreover, cluster analysis (CA) was utilized to create a dendrogram showing similarities and differences between genotypes.

\section{Results and discussion}

Some pomological properties of 10 jujube genotypes are shown in Table 1. There was a statistically significant difference in fruit weight, flesh/stone ratio, fruit width and length among genotypes $(\mathrm{P} \leq 0.05)$. In terms of the investigated characteristics, genotype 10 was the first, followed by genotype 9 . On the other hand, the values of genotype 8 were found to be low, in general. Differences in fruit weight $(2.73 \mathrm{~g}$ to $24.33 \mathrm{~g}$ ) caused a higher standard deviation. Thus, genotypes exhibited heterogeneity for this trait. The flesh/stone ratio ranged from 4.93 to 27.63. Similar to average fruit weight, higher standard deviation occurred. Minimum and maximum values of fruit width and length were determined as $17.55-34.83$ and $18.64-37.48 \mathrm{~mm}$, respectively. Jujube genotypes (Ziziphus mauritiana) with large fruits are reported to have a fruit weight range of $4 \mathrm{~g}$ (Gorh) to $36 \mathrm{~g}$ (Foladi) under the climatic conditions of Pakistan. The highest and lowest stone weight were found in the same genotype (Abbas et al., 2012). Confirming our results, fruit weight, width, and length were noticed as $23 \mathrm{~g}$, $37 \mathrm{~mm}$ and $40 \mathrm{~mm}$, respectively by Galindo et al. (2015). Furthermore, for three different jujube genotypes, Kavas and Dalkılıç (2015) explained the variation range of fruit weight $(8.21-28.85 \mathrm{~g})$, fruit width $(24.02-37.35 \mathrm{~mm})$ and fruit length $(29.47$ $43.73 \mathrm{~mm}$ ) in Kizılcaköy, Aydın. The change of data based on genotypes was also revealed in the earlier findings of Gao et al. (2012). These findings are synchronized with our study. 
Table 1. Pomological properties of jujube genotypes

\begin{tabular}{c|c|c|c|c|c}
\hline $\begin{array}{c}\text { Genotypes } \\
\text { number }\end{array}$ & $\begin{array}{c}\text { Fruit weight } \\
(\mathbf{g})\end{array}$ & $\begin{array}{c}\text { Stone weight } \\
(\mathbf{g})\end{array}$ & $\begin{array}{c}\text { Flesh/stone } \\
\text { ratio }\end{array}$ & $\begin{array}{c}\text { Fruit width } \\
(\mathbf{m m})\end{array}$ & $\begin{array}{c}\text { Fruit length } \\
(\mathbf{m m})\end{array}$ \\
\hline 1 & $5.37 \mathrm{c}$ & $0.78^{\mathrm{a}}$ & $5.88 \mathrm{f}$ & $22.62 \mathrm{~b}$ & $21.80 \mathrm{c}$ \\
2 & $4.72 \mathrm{e}$ & 0.41 & $10.47 \mathrm{c}$ & $19.17 \mathrm{~cd}$ & $19.88 \mathrm{def}$ \\
3 & $5.03 \mathrm{~d}$ & 0.76 & $5.62 \mathrm{~g}$ & $19.92 \mathrm{c}$ & $20.45 \mathrm{cde}$ \\
4 & $3.26 \mathrm{~h}$ & 0.32 & $9.19 \mathrm{~d}$ & $19.16 \mathrm{~cd}$ & $18.64 \mathrm{f}$ \\
5 & $4.31 \mathrm{f}$ & 0.64 & $5.74 \mathrm{fg}$ & $19.08 \mathrm{~cd}$ & $19.22 \mathrm{ef}$ \\
6 & $4.30 \mathrm{f}$ & 0.68 & $5.32 \mathrm{~h}$ & $18.17 \mathrm{~d}$ & $21.43 \mathrm{~cd}$ \\
7 & $3.59 \mathrm{~g}$ & 0.43 & $7.35 \mathrm{e}$ & $17.55 \mathrm{~d}$ & $18.95 \mathrm{ef}$ \\
8 & 2.731 & 0.46 & $4.93 \mathrm{r}$ & $17.68 \mathrm{~d}$ & $18.76 \mathrm{ef}$ \\
9 & $7.16 \mathrm{~b}$ & 0.55 & $12.00 \mathrm{~b}$ & $23.76 \mathrm{~b}$ & $25.68 \mathrm{~b}$ \\
10 & $24.33 \mathrm{a}$ & 0.85 & $27.63 \mathrm{a}$ & $34.83 \mathrm{a}$ & $37.48 \mathrm{a}$ \\
\hline Min. & 2.73 & 0.32 & 4.93 & 17.55 & 18.64 \\
Max. & 24.33 & 0.85 & 27.63 & 34.83 & 37.48 \\
Avg. & 6.48 & 0.59 & 9.41 & 21.19 & 22.23 \\
\hline St. dev. & 6.17 & 0.17 & 6.60 & 5.07 & 5.61 \\
\hline
\end{tabular}

The differences in the means were determined by the Duncan test according to $\mathrm{P} \leq 0.05$. ${ }^{\mathrm{a}}$ : Not significant

For all color variables, significant differences among genotypes occurred (Table 2). Generally, the highest values were obtained in genotype 6 , except $a^{*}$ value. In genotype 5 , the $a^{*}$ value, which expresses the red color, was the maximum. At the same time, it has a darker color owing to the $\mathrm{L}^{*}$ value is low. Moreover, the hue value was found low. Thus, this genotype has come to the fore in terms of color. The $b^{*}(5.62)$ and $h^{\circ}$ (5.71) values identified a high level of variation. As compared with Galindo et al. (2015), it was seen that $h^{\circ}$ value was lower in our study. This emphasizes that the red color is dominant. In contrast to this research, Kavas and Dalkıliç (2015) did not find any difference in this matter. The color values were influenced by many factors such as genotype, harvest time, cultural practices, crop load, etc. (Wang et al., 2012; Gündüz and Saraçoğlu, 2014).

Biochemical properties ( $\mathrm{pH}, \mathrm{TSS}$, TA, total phenol and antioxidant activity) significantly differed depending on genotypes (Table 3). No variation was observed in terms of $\mathrm{pH}$ and TA. The average value of $\mathrm{pH}$ was 5.11 and the TA value was $0.45 \%$. Genotypes are similar in this respect. In this trial, minimum TSS content was found to be $19.20 \%$ while a maximum of $45.34 \%$. The high standard deviation indicates a high variation in the population on account of phenol content and antioxidant activity. This diversity is desirable for plant breeders. Total phenol content and antioxidant activity were detected to be 239.90-322.39 mg GAE/g FW and 43.86-96.75 $\mu \mathrm{mol} \mathrm{TE/g} \mathrm{FW,}$ respectively. Jujube contains much higher TSS than many fruit species (Yao, 2013). While the TTS content of jujube fruits was determined as 24\% (Abbas et al., 2012), 32\% (Kavas and Dalkılıç, 2015), 19\% (Koley et al., 2016), 30\% (Gao et al., 2012) and $32 \%$ (Ecevit et al., 2008) in previous research, this value was found to be extremely high as $45.34 \%$ by ours. Godi and Joshi (2016) stated that the variation in TSS among the jujube genotype may vary mainly due to different harvest periods or genotype. As it is known, if the product load decreases, TSS and TA values increase but not the $\mathrm{pH}$. Compared with the previous study (Galindo et al., 2015), TA content was found to be lower, $\mathrm{pH}$ was similar in our study. It is pointed out that jujube fruits are rich in total 
phenol contents and antioxidant activity (Yao, 2013). In Indian jujube commercial cultivars (Z. mauritiana), the range of total phenol was 172-328.6 mg GAE/100 g (Koley et al., 2016). Our findings are supported by this study. Many external factors may influence the change of the phenol content (Imamoglu, 2016). Although fruits such as strawberry (Zheng et al., 2007), grapes (İşçi et al., 2014), black mulberry (Özgen et al., 2009) contain high phenol, Ziziphus contains much more phenol than these fruit species. When compared to cherry and grapefruits with high antioxidant activity, jujube genotypes are superior in this respect (Çağlar and Demirci, 2017; Beyhan et al., 2018).

Table 2. Color values of jujube genotypes

\begin{tabular}{c|c|c|c|c|c}
\hline $\begin{array}{c}\text { Genotypes } \\
\text { number }\end{array}$ & $\mathbf{L}^{*}$ & $\mathbf{a}^{*}$ & $\mathbf{b}^{*}$ & $\mathbf{C}^{*}$ & $\mathbf{h}^{\circ}$ \\
\hline 1 & $37.54 \mathrm{e}$ & $25.94 \mathrm{ef}$ & $31.46 \mathrm{e}$ & $40.78 \mathrm{~d}$ & $50.49 \mathrm{c}$ \\
2 & $36.40 \mathrm{f}$ & $26.67 \mathrm{de}$ & $30.71 \mathrm{e}$ & $40.66 \mathrm{~d}$ & $49.03 \mathrm{~d}$ \\
3 & $42.94 \mathrm{~b}$ & $27.34 \mathrm{c}$ & $38.55 \mathrm{~b}$ & $47.24 \mathrm{~b}$ & $54.56 \mathrm{a}$ \\
4 & $40.24 \mathrm{c}$ & $26.95 \mathrm{~d}$ & $33.22 \mathrm{~d}$ & $42.78 \mathrm{c}$ & $50.95 \mathrm{c}$ \\
5 & $33.40 \mathrm{~g}$ & $30.27 \mathrm{a}$ & $22.55 \mathrm{~h}$ & $37.75 \mathrm{e}$ & $36.68 \mathrm{f}$ \\
6 & $45.70 \mathrm{a}$ & $28.63 \mathrm{~b}$ & $41.58 \mathrm{a}$ & $50.48 \mathrm{a}$ & $55.45 \mathrm{a}$ \\
7 & $38.53 \mathrm{~d}$ & $21.56 \mathrm{~h}$ & $26.46 \mathrm{~g}$ & $34.13 \mathrm{f}$ & $50.83 \mathrm{c}$ \\
8 & $33.78 \mathrm{~g}$ & $30.05 \mathrm{a}$ & $26.34 \mathrm{~g}$ & $39.96 \mathrm{~d}$ & $41.24 \mathrm{e}$ \\
9 & $37.36 \mathrm{e}$ & $24.98 \mathrm{~g}$ & $28.67 \mathrm{f}$ & $38.03 \mathrm{e}$ & $48.93 \mathrm{~d}$ \\
10 & $38.92 \mathrm{~d}$ & $25.54 \mathrm{fg}$ & $34.18 \mathrm{c}$ & $42.67 \mathrm{c}$ & $53.23 \mathrm{~b}$ \\
\hline Min. & 33.40 & 21.56 & 22.55 & 34.13 & 36.68 \\
Max. & 45.70 & 30.27 & 41.58 & 50.48 & 55.45 \\
Avg. & 38.48 & 26.79 & 31.37 & 41.45 & 49.14 \\
\hline St. dev. & 3.71 & 2.55 & 5.62 & 4.59 & 5.71 \\
\hline
\end{tabular}

The differences in the means were determined by the Duncan test according to $\mathrm{P} \leq 0.05$

Table 3. Some biochemical properties of jujube genotypes

\begin{tabular}{c|c|c|c|c|c}
\hline $\begin{array}{c}\text { Genotypes } \\
\text { number }\end{array}$ & $\mathbf{p H}$ & $\begin{array}{c}\text { TSS } \\
(\boldsymbol{\%})\end{array}$ & $\begin{array}{c}\text { TA } \\
(\boldsymbol{\%})\end{array}$ & $\begin{array}{c}\text { Total phenol } \\
(\mathbf{m g ~ G A E} / \mathbf{g} \text { fw) }\end{array}$ & $\begin{array}{c}\text { Antioxidant } \\
\text { activity } \\
(\boldsymbol{\mu m o l ~ T E / g ~ f w ) ~}\end{array}$ \\
\hline 1 & $5.03 \mathrm{~d}$ & $20.67 \mathrm{~d}$ & $0.50 \mathrm{c}$ & $259.13 \mathrm{ab}$ & $96.75 \mathrm{a}$ \\
2 & $4.97 \mathrm{~d}$ & $22.81 \mathrm{~cd}$ & $0.58 \mathrm{~b}$ & $251.59 \mathrm{ab}$ & $71.73 \mathrm{ab}$ \\
3 & $4.76 \mathrm{e}$ & $23.47 \mathrm{~cd}$ & $0.71 \mathrm{a}$ & $253.87 \mathrm{ab}$ & $75.59 \mathrm{ab}$ \\
4 & $5.27 \mathrm{~b}$ & $28.94 \mathrm{bc}$ & $0.49 \mathrm{c}$ & $239.90 \mathrm{~b}$ & $63.38 \mathrm{bc}$ \\
5 & $5.58 \mathrm{a}$ & $30.00 \mathrm{~b}$ & $0.36 \mathrm{~d}$ & $242.33 \mathrm{~b}$ & $53.29 \mathrm{bc}$ \\
6 & $4.71 \mathrm{e}$ & $19.20 \mathrm{~d}$ & $0.71 \mathrm{a}$ & $322.39 \mathrm{a}$ & $73.52 \mathrm{ab}$ \\
7 & $5.16 \mathrm{c}$ & $25.20 \mathrm{bcd}$ & $0.34 \mathrm{~d}$ & $241.52 \mathrm{~b}$ & $74.68 \mathrm{ab}$ \\
8 & $5.22 \mathrm{bc}$ & $45.34 \mathrm{a}$ & $0.35 \mathrm{~d}$ & $247.56 \mathrm{ab}$ & $67.24 \mathrm{bc}$ \\
9 & $5.21 \mathrm{bc}$ & $24.00 \mathrm{bcd}$ & $0.34 \mathrm{~d}$ & $206.04 \mathrm{~b}$ & $43.86 \mathrm{c}$ \\
10 & $5.22 \mathrm{bc}$ & $22.67 \mathrm{~cd}$ & $0.17 \mathrm{e}$ & $237.99 \mathrm{~b}$ & $54.91 \mathrm{bc}$ \\
\hline Min. & 4.71 & 19.20 & 0.17 & 239.90 & 43.86 \\
Max. & 5.58 & 45.34 & 0.71 & 322.39 & 96.75 \\
Avg. & 5.11 & 26.23 & 0.45 & 250.23 & 67.50 \\
\hline St. dev. & 0.25 & 7.76 & 0.17 & 43.24 & 18.32 \\
\hline
\end{tabular}

The differences in the means were determined by the Duncan test according to $\mathrm{P} \leq 0.05$ 
Results presented in Table 4 display the organoleptic features of jujube genotypes. The difference between genotypes was statistically significant for flavor (1.00 to 9.00). The best flavor has been identified in genotypes 10 and 7 , and there were also remarkable by texture. Considering general acceptability, genotypes 10 (9.00) and 8 (1.67) ranked first and last, respectively. The flavor of fruit was generally described as apple by Kavas and Dalkılıç (2015). It was reported that the product load and fruit flavor were not related to Grande de Albatera variety (Galindo et al., 2015). The variation observed in these characteristics was also demonstrated in 19 genotypes evaluated by Godi and Joshi (2016). In addition to fresh consumption, fruits are also evaluated in different forms such as gel, pickle, and jelly. For this purpose, sensory evaluations are also important (Uddin and Hussain, 2012).

Table 4. Organoleptic evaluation of jujube genotypes

\begin{tabular}{c|c|c|c}
\hline Genotypes number & Flavor & Texture & General acceptability \\
\hline 1 & $1.00 \mathrm{e}$ & $6.33 \mathrm{ab}$ & $5.67 \mathrm{bc}$ \\
2 & $5.67 \mathrm{bc}$ & $4.33 \mathrm{bc}$ & $4.33 \mathrm{~cd}$ \\
3 & $3.00 \mathrm{~d}$ & $4.33 \mathrm{bc}$ & $5.00 \mathrm{c}$ \\
4 & $6.33 \mathrm{bc}$ & $4.33 \mathrm{bc}$ & $2.33 \mathrm{de}$ \\
5 & $7.00 \mathrm{~b}$ & $3.67 \mathrm{c}$ & $5.67 \mathrm{bc}$ \\
6 & $5.00 \mathrm{c}$ & $4.33 \mathrm{bc}$ & $2.33 \mathrm{de}$ \\
7 & $9.00 \mathrm{a}$ & $6.33 \mathrm{ab}$ & $2.33 \mathrm{de}$ \\
8 & $7.00 \mathrm{~b}$ & $3.67 \mathrm{c}$ & $1.67 \mathrm{e}$ \\
9 & $5.00 \mathrm{c}$ & $6.33 \mathrm{ab}$ & $7.67 \mathrm{ab}$ \\
10 & $9.00 \mathrm{a}$ & $7.00 \mathrm{a}$ & $9.00 \mathrm{a}$ \\
\hline Min. & 1.00 & 3.67 & 1.67 \\
Max. & 9.00 & 7.00 & 9.00 \\
Avg. & 5.80 & 5.07 & 4.53 \\
\hline St. dev. & 2.49 & 1.53 & 2.60 \\
\hline
\end{tabular}

The differences in the means were determined by the Duncan test according to $\mathrm{P} \leq 0.05$. Flavor: no taste (1), elaeagnus-apple (3), apple-pear (5), elaeagnus (7), apple (9); Texture: soft (3), medium (5), hard (7); General acceptability: poor (1), fair (3), medium (5), good (7), very good (9)

The correlation coefficients between the features examined of jujube genotypes are seen in Table 5. Accordingly, the highest correlation was determined between fruit width and length $(\mathrm{r}=0.976 ; \mathrm{p}<0.01)$. Further, fruit width and length showed a significantly positive and strong correlation to fruit weight $(r=0.960$ and 0.968 ; $\mathrm{p}<0.01)$, stone weight $(\mathrm{r}=0.591$ and $0.589 ; \mathrm{p}<0.01)$ and flesh/stone ratio $(\mathrm{r}=0.918$ and $0.923 ; p<0.01)$. Fruit weight had a positive correlation with stone weight and flesh/stone ratio but a negative correlation with total acidity. $\mathrm{L}^{*}, \mathrm{~b}^{*}, \mathrm{~h}^{\circ}$ and $\mathrm{C}^{*}$ color of the fruit were also found to be correlated. As $\mathrm{L}^{*}$ increased, $\mathrm{b}^{*}(\mathrm{r}=0.91), \mathrm{h}^{\circ}(\mathrm{r}=0.780)$, $\mathrm{C}^{*}(\mathrm{r}=0.861)$ and total acidity $(\mathrm{r}=0.592)$ increased; however, $\mathrm{pH}(\mathrm{r}=-0.757)$ and TSS $(r=-0.556)$ decreased. Fruit $a^{*}$ value showed a positive correlation to $h^{\circ}$ value and TSS, but negative correlation to $\mathrm{C}^{*}$ value. A positive correlation was observed between fruit $b^{*}$ color and total acidity, stone weight while $\mathrm{pH}$ negatively affected TSS. Fruit $\mathrm{h}^{\circ}$ color had a positive and strong correlation with total acidity $(\mathrm{r}=0.659)$ but weak correlation with stone weight $(\mathrm{r}=0.404)$. Fruit $\mathrm{C}^{*}$ value showed a positive correlation 
to total acidity $(\mathrm{r}=0.392 ; \mathrm{p}<0.05)$, but negative correlation to $\mathrm{pH}(\mathrm{r}=0.754 ; \mathrm{p}<0.01)$ and TSS $(\mathrm{r}=0.653 ; \mathrm{p}<0.01)$. A negative correlation was observed between antioxidant activity and $\mathrm{pH}(\mathrm{r}=-0.474 ; \mathrm{p}<0.01)$. Total phenol had a positive correlation with $\mathrm{L}^{*}$ $(\mathrm{r}=0.407 ; \mathrm{p}<0.05), \mathrm{b}^{*}(\mathrm{r}=0.412 ; \mathrm{p}<0.05), \mathrm{h}^{\circ}(\mathrm{r}=0.468 ; \mathrm{p}<0.01)$ and total acidity $(\mathrm{r}=0.431 ; \mathrm{p}<0.05)$. From the other side, a negative correlation appeared between total phenol and $\mathrm{pH}(\mathrm{r}=-0.436 ; \mathrm{p}<0.05)$. In jujube with an apple-like flavor; $\mathrm{pH}$ and flesh/stone ratio were higher, whereas $h^{\circ}$ value, total acidity and antioxidant activity decreased. In jujube with hard flesh; fruit weight, flesh/stone ratio, fruit width and length were higher, whereas color $\mathrm{a}^{*}$ was lower than soft flesh. Fruit acceptability had a positive and strong correlation with fruit and stone weight, fruit width and length, flesh/stone ratio and texture while weak, and negative correlation with TSS. Positive correlation between phenolic content and antioxidant activity is mentioned (Gao et al., 2012; Imamoglu, 2016; Koley et al., 2016) however, a correlation was not found in our results. This is also confirmed by Li et al. $(2005,2007)$.

Principal component analysis (PCA) defined $88.850 \%$ of the genotypes with 18 properties and was explained by four principal components (Table 6). PC1, PC2, PC3 and PC4 accounted for $30.905 \%, 30.703 \%, 14.883 \%$ and $12.359 \%$ respectively of the variability. Consequently, PC1 constituted mainly fruit width, length, weight, acceptability, flesh/stone ratio and stone weight; PC2 included mainly ground color $\mathrm{b}^{*}$, $\mathrm{L}^{*}, \mathrm{~h}^{\circ}, \mathrm{C}^{*}, \mathrm{pH}$, antioxidant activity, and total acidity; PC3 show mainly ground color $\mathrm{a}^{*}$, texture and TSS; PC4 represents mainly flavor and total phenol.

Figure 2 shows biplot based on PCA for fruit quality traits in jujube genotypes in rotated space. PC1 explained fruit width, length, weight, acceptability, flesh/stone ratio, and stone weight. Positive values for PC2 indicated ground color $b^{*}, \mathrm{~L}^{*}, \mathrm{~h}^{\circ}, \mathrm{C}^{*}$, antioxidant activity, and total acidity, while negative PC2 values display $\mathrm{pH}$. Positive values for PC3 represent ground color $\mathrm{a}^{*}$ and TSS, while negative PC3 values point out the texture. PCA was performed to study the correlation between fruit quality parameters (Koley et al., 2016).

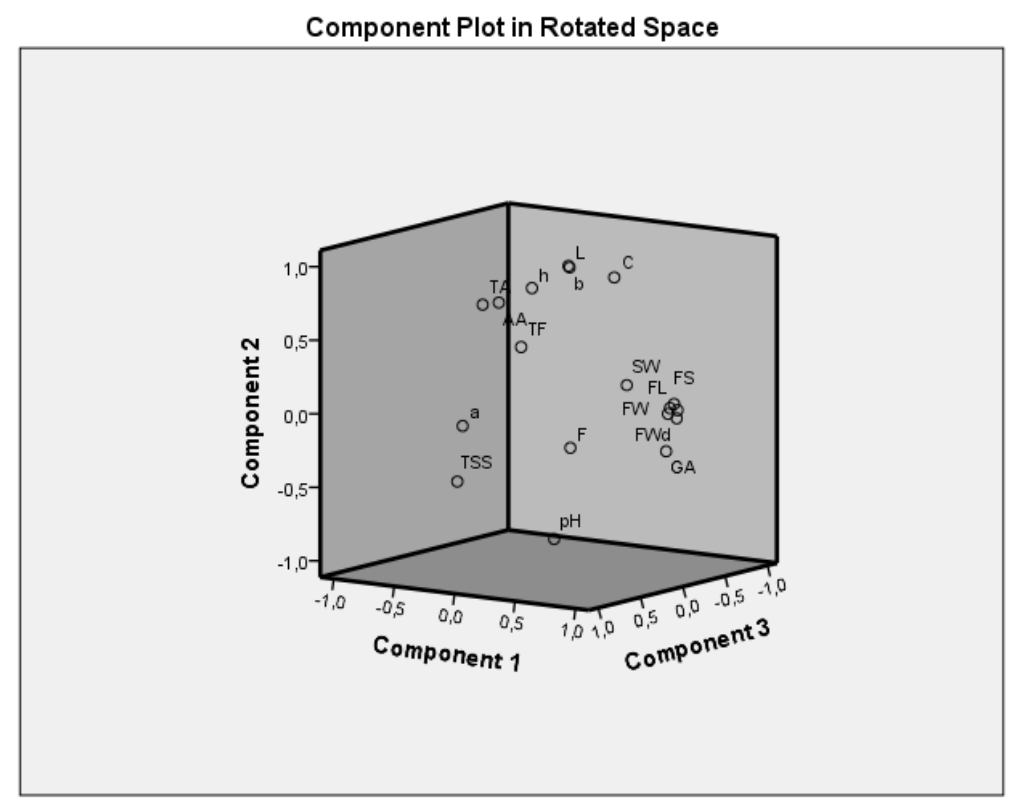

Figure 2. Biplot based on principal components analysis (PCA) for fruit quality traits in rotated space 
Table 5. Pearson correlation coefficients among traits of jujube genotypes

\begin{tabular}{|c|c|c|c|c|c|c|c|c|c|c|c|c|c|c|c|c|c|}
\hline & FW & SW & FS & FWd & FL & $\mathbf{L}$ & $\mathbf{a}$ & b & h & C & pH & TSS & TA & TF & $\mathbf{A A}$ & $\mathbf{F}$ & $\mathbf{T}$ \\
\hline SW & $0.577 * *$ & & & & & & & & & & & & & & & & \\
\hline FS & $0.952^{* *}$ & 0.318 & & & & & & & & & & & & & & & \\
\hline FWd & $0.960^{* * *}$ & $0.591^{* * *}$ & $0.918^{* *}$ & & & & & & & & & & & & & & \\
\hline FL & $0.968^{* *}$ & $0.589^{* *}$ & $0.923^{* *}$ & $0.976^{* *}$ & & & & & & & & & & & & & \\
\hline $\mathbf{L}$ & 0.056 & 0.267 & -0.006 & 0.004 & 0.090 & & & & & & & & & & & & \\
\hline $\mathbf{a}$ & -0.213 & 0.104 & -0.316 & -0.242 & -0.244 & -0.127 & & & & & & & & & & & \\
\hline b & 0.188 & $0.385^{*}$ & 0.107 & 0.152 & 0.217 & $0.921^{* *}$ & 0.046 & & & & & & & & & & \\
\hline $\mathbf{h}$ & 0.088 & $0.404^{*}$ & -0.033 & 0.042 & 0.102 & $0.780^{* *}$ & $0.435^{*}$ & $0.917^{* *}$ & & & & & & & & & \\
\hline C & 0.282 & 0.261 & 0.273 & 0.263 & 0.316 & $0.861^{* *}$ & $-0.473^{* *}$ & $0.846^{* *}$ & $0.566^{* *}$ & & & & & & & & \\
\hline pH & 0.111 & -0.248 & 0.189 & 0.134 & 0.054 & $-0.757^{* *}$ & 0.083 & $-0.819^{* *}$ & $-0.700^{* * *}$ & $-0.754^{* * *}$ & & & & & & & \\
\hline TSS & -0.258 & $-0.406^{*}$ & -0.228 & -0.266 & -0.291 & $-0.556^{* *}$ & $0.416^{*}$ & $-0.508^{* *}$ & \begin{tabular}{|l|l} 
& -0.296
\end{tabular} & $-0.653^{* *}$ & $0.465^{* *}$ & & & & & & \\
\hline $\mathbf{T A}$ & $-0.539^{* * *}$ & 0.000 & $-0.589^{* *}$ & $-0.542^{* *}$ & $-0.517^{* *}$ & $0.592^{* *}$ & 0.242 & $0.621^{* *}$ & $0.659^{* *}$ & $0.392^{*}$ & $-0.731^{* *}$ & -0.294 & & & & & \\
\hline $\mathbf{T F}$ & -0.131 & 0.159 & -0.225 & -0.187 & -0.120 & $0.407^{*}$ & 0.229 & $0.412^{*}$ & $0.468^{* *}$ & 0.223 & $-0.436^{*}$ & -0.190 & $0.431^{*}$ & & & & \\
\hline $\mathbf{A A}$ & -0.258 & 0.113 & -0.343 & -0.212 & -0.263 & 0.202 & -0.087 & 0.233 & 0.171 & 0.257 & $-0.474^{* *}$ & -0.133 & 0.338 & 0.210 & & & \\
\hline $\mathbf{F}$ & 0.335 & -0.310 & $0.438^{*}$ & 0.172 & 0.246 & -0.241 & -0.162 & -0.359 & $-0.382^{*}$ & -0.228 & $0.490^{* *}$ & 0.307 & $-0.590^{* *}$ & -0.137 & $-0.427^{*}$ & & \\
\hline $\mathbf{T}$ & $0.492^{* *}$ & 0.293 & $0.489^{* *}$ & $0.544^{* *}$ & $0.537^{* *}$ & 0.093 & $-0.592^{* *}$ & 0.036 & -0.217 & 0.350 & -0.001 & -0.310 & -0.336 & -0.083 & -0.096 & 0.058 & \\
\hline GA & $0.691^{* *}$ & $0.587^{* *}$ & $0.638^{* *}$ & $0.749^{* *}$ & $0.708^{* *}$ & -0.114 & -0.140 & -0.017 & -0.074 & 0.065 & 0.180 & $-0.379^{*}$ & -0.358 & -0.202 & -0.352 & -0.034 & $0.493^{* *}$ \\
\hline
\end{tabular}

$*$ Significant at $\mathrm{P}<0.05, * *$ Significant at $\mathrm{P}<0.01$, ns: Non-significance

FW: fruit weight; SW: stone weight; FS: flesh/stone ratio; FWd: fruit width; FL: fruit length; L: L*; a: a*; b: b*; h: hº C: $\mathrm{C}^{*}$; pH; TSS: total solids soluble; TA: total acidity; TF: total phenol; AA: antioxidant activity; F: flavor; T: texture; GA: general acceptability 
Table 6. Component loading in principle component analysis (PCA)

\begin{tabular}{c|c|c|c|c}
\hline Traits & PC1 & PC2 & PC3 & PC4 \\
\hline W & $\mathbf{0 . 9 5 6}$ & -0.004 & -0.150 & 0.141 \\
L & $\mathbf{0 . 9 4 2}$ & 0.094 & -0.137 & 0.240 \\
FW & $\mathbf{0 . 9 3 3}$ & 0.070 & -0.101 & 0.280 \\
A & $\mathbf{0 . 8 7 4}$ & -0.235 & -0.139 & -0.163 \\
FS & $\mathbf{0 . 8 4 1}$ & 0.006 & -0.210 & 0.434 \\
SW & $\mathbf{0 . 7 3 4}$ & 0.239 & 0.124 & -0.461 \\
Gb & 0.178 & $\mathbf{0 . 9 6 5}$ & 0.006 & -0.122 \\
GL & 0.028 & $\mathbf{0 . 9 3 2}$ & -0.193 & -0.047 \\
Gh & 0.141 & $\mathbf{0 . 8 7 8}$ & 0.398 & -0.170 \\
pH & 0.136 & $\mathbf{- 0 . 8 6 8}$ & 0.129 & 0.277 \\
GC & 0.187 & $\mathbf{0 . 8 2 5}$ & -0.508 & -0.018 \\
AA & -0.204 & $\mathbf{0 . 7 3 0}$ & 0.296 & -0.209 \\
TA & -0.448 & $\mathbf{0 . 6 6 9}$ & 0.140 & -0.494 \\
Ga & -0.022 & 0.009 & $\mathbf{0 . 9 8 4}$ & -0.128 \\
T & 0.497 & -0.091 & $\mathbf{- 0 . 8 1 4}$ & -0.175 \\
TSS & -0.368 & -0.466 & $\mathbf{0 . 5 5 4}$ & 0.414 \\
F & 0.163 & -0.267 & -0.024 & $\mathbf{0 . 8 3 3}$ \\
TF & -0.355 & 0.344 & -0.182 & $\mathbf{- 0 . 5 6 4}$ \\
Eigenvalue & $\mathbf{5 . 5 6 3}$ & $\mathbf{5 . 5 2 7}$ & $\mathbf{2 . 6 7 9}$ & $\mathbf{2 . 2 2 5}$ \\
Proportion $(\%)$ & $\mathbf{3 0 . 9 0 5}$ & $\mathbf{3 0 . 7 0 3}$ & $\mathbf{1 4 . 8 8 3}$ & $\mathbf{1 2 . 3 5 9}$ \\
Cumulative $(\%)$ & $\mathbf{3 0 . 9 0 5}$ & $\mathbf{6 1 . 6 0 8}$ & $\mathbf{7 6 . 4 9 1}$ & $\mathbf{8 8 . 8 5 0}$ \\
\hline
\end{tabular}

Extraction method: principal component analysis. Rotation method: Varimax with Kaiser normalization

Clustering analysis (CA) was used to determine the degree of similarity of jujube genotypes, is shown in Figure 3 as dendrograms. Thus, the genotypes were categorized under two main groups. $6^{\text {th }}$ genotype formed a separate group from the others. The similarities or differences among the jujube genotypes examined with CA showed a correlation with those examined with PCA in terms of investigated characteristics.
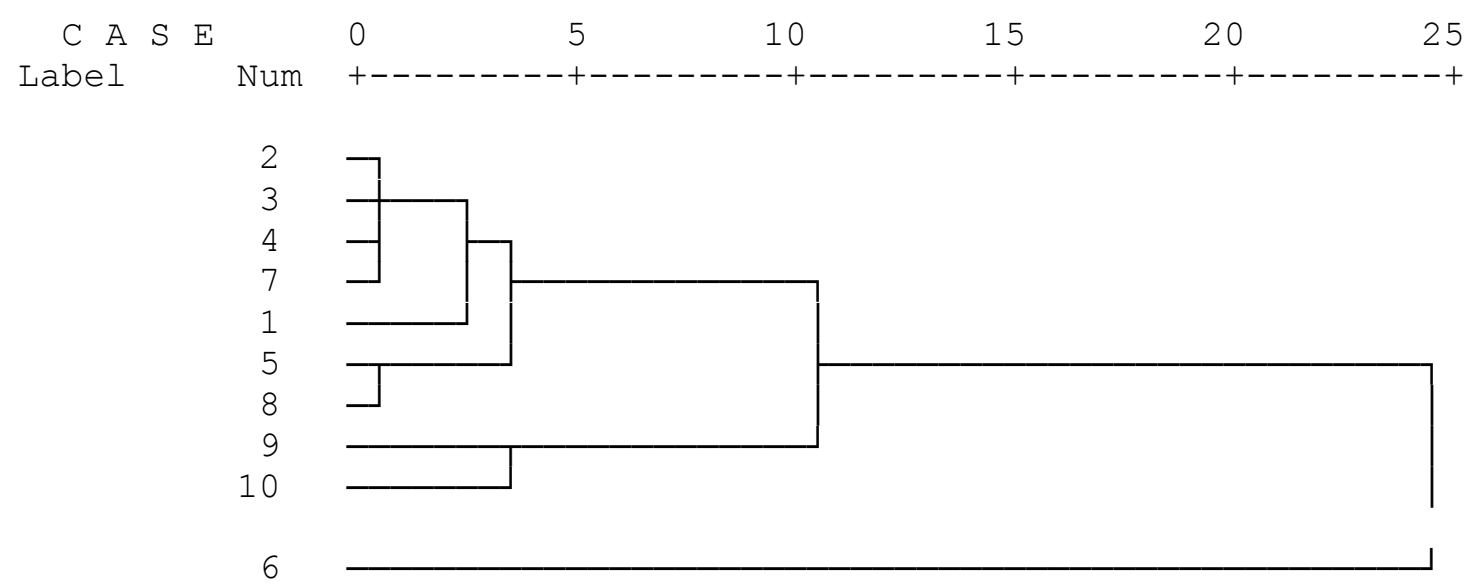

Figure 3. Dendrogram of hierarchical cluster analysis obtained by Ward's clustering method 


\section{Conclusion}

This study was planned to evaluate some fruit characteristics of 10 jujube genotypes collected from the Demirci location in Turkey. In terms of TSS content, especially genotype 8 is very important and this value is also high in genotype 5. Besides, the fruits of these two genotypes are dark red, high phenol content, elaeagnus taste, and soft texture. Genotype 1 is prominent because of its rich antioxidant content. In this respect, it is a natural antioxidant source and can be considered as an alternative healthy food. On the other hand, genotype 6 is a different group from other genotypes due to its high phenol content and light color. Also, the fruits of genotype 10 are large and taste like apple and their acceptability is good. The jujubes mentioned above are suitable for table consumption. They seem to have a beneficial effect on folk medicine due to their phytochemical contents. The identified genotypes show variation under the same ecological conditions. In this context, they are valuable as a genetic resource. These genotypes are promising. In future studies, a patent may be granted for any genotypes. They can also be used as parents in breeding programs. Furthermore, molecular studies can be performed to determine the genetic relationship. For jujube, this study is essential because there is little information in Turkey. It is important to continue such studies in terms of evaluating the present population in nature and identifying genotypes for different purposes.

\section{REFERENCES}

[1] Abbas, M. M., Sharif, N., Mohar, T. A. (2012): Quality evaluation of promising ber (Zizyphus mauritiana) varieties under climatic conditions of Faisalabad. - J. Agric. Res. 50(3): 401-409.

[2] Abdel-Zaher, A. O., Salim, S. Y., Assaf, M. H., Abdel Hady, R. H. (2005): Anti diabetic activity and toxicity of Zizyphus spina-christi leaves. - Journal of Ethnopharmacology 101(1-3): 129-138.

[3] Ahmad, R., Malik, W., Anjum, M. A. (2019): Genetic diversity and selection of suitable molecular markers for characterization of indigenous Zizyphus germplasm. - ErwerbsObstbau 61: 345-353.

[4] Anşin, R., Özkan, Z. C. (1997): Tohumlu Bitkiler: (Spermatophyta) Odunsu Taksonlar. Karadeniz Teknik Üniversitesi Basımevi, Trabzon, pp. 465-466 (in Turkish).

[5] Benzie, I. F. F., Strain, J. J. (1996): Ferric reducing ability of plasma (FRAP) as a measure of antioxidant power: the FRAP assay. - Anal. Biochem. 239: 70-76.

[6] Beyhan, Ö., Demir, T., Yurt, B. (2018): Determination of antioxidant activity, phenolic compounds and biochemical properties of cherry laurel (Laurocerasus officinalis R.) grown in Sakarya/Turkey. - Bahçe 47(1): 17-22.

[7] Çağlar, M. Y., Demirci, M. (2017): Üzümsü Meyvelerde Bulunan Fenolik Bileşikler ve Beslenmedeki Önemi. - Avrupa Bilim ve Teknoloji Dergisi Cilt. 7(11): 18-26 (in Turkish).

[8] Chen, X., Chen, R., Wang, Y., Wu, C., Huang, J. (2019): Genome-wide identification of WRKY transcription factors in Chinese jujube (Ziziphus jujuba Mill.) and their involvement in fruit developing, ripening, and abiotic stress. - Genes. 10: 360. DOI: 10.3390/genes10050360.

[9] Choi, S. H., Ahn, J. B., Kozukue, N., Levin, C. E., Friedman, M. (2011): Distribution of free amino acids, flavonoids, total phenolics, and antioxidative activities of jujube (Ziziphus jujuba) fruits and seeds harvested from plants grown in Korea. - Journal of Agricultural and Food Chemistry 59: 6594-6604. 
[10] Dahlia, F., Benito, C., Boussaid, M. (2019): Genetic diversity of fruits in wild jujube (Ziziphus lotus L. Desf.) natural populations from Algeria. - Agriculture \& Forestry 65(1): 165-183. DOI: 10.17707/AgricultForest.65.1.17.

[11] Ecevit, M. F., Hallaç, F., Dilmaç Ünal, T. (2002): Denizli ili Çivril İlçesi Gümüşsu Yöresinde Yetişmekte Olan Ünnap (Ziziphus jujuba Mill.)'ın Seleksiyon Yoluyla Islahı Üzerinde Araştırmalar. - Tübitak Togtag Tarp-1988, Ankara (in Turkish).

[12] Ecevit, M. F., Şan, B., Dilmaç, Ünal., T., Hallaç., Türk, F., Yıldırım, A. N., Polat, M., Y1ldırım, F. (2008): Selection of superior ber (Ziziphus jujuba L.) genotypes in çivril region. - Tarım Bilimleri Dergisi 14(1): 51-56. (in Turkish).

[13] Galindo, A., Noguera-Artiaga, L., Cruz, Z. N., Burlo, F., Hernandez, F., Torrecillas, A., Carbonell-Barrachina, A. A. (2015): Sensory and physico-chemical quality attributes of jujube fruits as affected by crop load. - LWT - Food Science and Technology 63: 899905.

[14] Gao, Q. H., Wu, C. S., Yu, J. G., Wang, M., Ma, Y. J., Li, C. L. (2012): Textural characteristic, antioxidant activity, sugar, organic acid, and phenolic profiles of 10 promising jujube (Ziziphus jujuba Mill.) selections. - Journal of Food Science 77(11): 1218-1225.

[15] Godi, N. F., Joshi, V. R. (2016): Studies on biochemical and organoleptic characters of different ber (Zizyphus mauritiana Lamk) genotypes. - Advances in Life Sciences 5(6).

[16] Gozlekci, S., Zarifi Khosroshahi, M., Kafkas, E. (2015): Some physical and chemical properties of two jujube (Zızıphus jujuba Mill.) genotypes grown in western Turkey. DOI: 10.17660/ActaHortic.2015.1074.11.

[17] Gündüz, K., Saraçoğlu, O. (2014): Changes in chemical composition, total phenolic content and antioxidant activities of jujube (Ziziphus jujuba Mill.) fruits at different maturation stages. - Acta Sci. Pol., Hortorum Cultus 13(2): 187-195.

[18] Imamoglu, H. (2016): Total antioxidant capacity, phenolic compounds and sugar content of Turkey Ziziphus jujubes. - Acta Sci. Pol. Hortorum Cultus 15(5): 93-108.

[19] İşçi, B., Şen, F., Güçlü Özdemir, A., Kaçar, E., Altun, A. (2014): Effects of modified atmosphere packing (MAP) and cold treatment on organically grown table grape cultivars. - Journal of Agriculture Faculty of Ege University 51(2): 191-199.

[20] İslam, M. B., Simmons, M. P. (2006): A thorny dilemma: testing alternative intrageneric classifications within Ziziphus (Rhamnaceae). - Sistematic Botany 31: 826842.

[21] Kamiloglu, O., Ericisli, S., Sengul, M., Toplu, C., Serce, S. (2009): Total phenolics and antioxidant activity of jujube (Zizyphus jujuba Mill.) genotypes selected from Turkey. Afr. J. Biotechnol. 8: 303-307.

[22] Karaçalı, İ. (2012): Bahçe Ürünlerinin Muhafaza ve Pazarlanması. - Ege Üniversitesi Ziraat Fakültesi Yayınları Bornova, İzmir (in Turkish).

[23] Karıncal1, M. (2003): Ziziphus jujuba Mill. (Hünnap) Bitkisinin Morfolojik, Anatomik, Ekolojik ve Polen Özelliklerinin Araştırılması, - Pamukkale Üniversitesi Fen Bilimleri enstitüsü Biyoloji Anabilim Dalı, Yüksek Lisans Tezi, Denizli (in Turkish).

[24] Kavas, İ., Dalkılıç, Z. (2015): Bazı Hünnap Genotiplerinin Morfolojik, Fenolojik Ve Pomolojik Özelliklerinin Belirlenmesi ve Melezleme Olanaklarının Araştırılması. Journal of Adnan Menderes University Agricultural Faculty 12(1): 57-72 (in Turkish).

[25] Koley, T. K., Kaur, C., Nagal, S., Walia, S., Jaggi, S. (2016): Antioxidant activity and phenolic content in genotypes of Indian jujube (Zizyphus mauritiana Lamk.). - Arabian Journal of Chemistry 9: S1044-S1052. http://dx.doi.org/10.1016/j.arabjc.2011.11.005.

[26] Li, J. W., Ding, S., Ding, X. (2005): Comparison of antioxidant capacities of extracts from five cultivars of Chinese jujube. - Process Biochem. 40: 3607-3613.

[27] Li, J. W., Fan, L. P., Ding, S. D., Ding, X. L. (2007): Nutritional composition of five cultivars of Chinese jujube. - Food Chem. 100(2): 454-460.

[28] Liu, M. (2006): Chinese jujube: botany and horticulture. - Horticultural Reviews 32: 229298. 
[29] Meligaared, M., Civille, G. V., Carr, B. T. (1991): Sensory Evaluation Techniques. 2nd Ed. - CRC Press, Boca Raton, FL.

[30] Mukhtar, H. M., Ansari, S. H., Ali, M., Naved, T. (2004): New compounds from Zizyphus vulgaris. - Pharmaceutical Biology 42(7): 508-511.

[31] Özgen, M., Serçe, S., Kaya, C. (2009): Phytochemical and antioxidant properties of anthocyanin Morus nigra and Morus rubra fruits. - Scientia Horticulturae 119: 275-279.

[32] Sharifa, N., Jaskania, M. J., Naqvia, S. A., Awa, F. S. (2019): Exploitation of diversity in domesticated and wild ber (Ziziphus mauritiana Lam.) germplasm for conservation and breeding in Pakistan. - Scientia Horticulturae 249: 228-239.

[33] Swain, T., Hillis, W. E. (1959): The phenolic constituents of Prunus domestica I.- the quantitative analysis of phenolic constituents. - J Sci Food Agric 10: 63-68.

[34] Thaipong, K., Boonprakob, U., Crosby, K., Cisneros-Zevallos, L., Byrne, D. H. (2006): Comparison of ABTS, DPPH, FRAP, and ORAC assays for estimating antioxidant activity from guava fruit extracts. - J Food Composit Anal 19: 669-675.

[35] Uddin, M. B., Hussai, I. (2012): Development of diversified technology for jujube (Ziziphus jujuba L) processing and preservation. - World Journal of Dairy \& Food Sciences 7(1): 74-78. DOI: 10.5829/idosi.wjdfs.2012.7.1.62115.

[36] Wang, H., Chen, F., Yang, H., Chen, Y., Zhang, L., An, H. (2012): Effects of ripening stage and cultivar on physicochemical properties and pectin nanostructures of jujubes. Carbohydrate Polymers 89: 1180-1188.

[37] Wojdylo, A., Carbonell-Barrachina, A. A., Legua, P., Hernandez, F. (2016): Phenolic composition, ascorbic acid content, and antioxidant capacity of Spanish jujube (Ziziphus jujube Mill.) fruits. - Food Chemistry 201: 307-314.

[38] Yao, S. (2013): Past, present, and future of jujubes - Chinese dates in the United States. HortScience 48(6): 672-680.

[39] Yaşa, F. (2016): Türkiye'de Yetiştirilen Hünnap Meyvesinin Bileşimi Ve Meyvenin Kurutulması Sırasında Bileşiminde Meydana Gelen Değişimler. - Pamukkale Üniversitesi Fen Bilimleri Enstitüsü Gıda Mühendisliği Anabilim Dalı Gıda Teknolojisi Bilim Dalı. Yüksek Lisans Tezi, Denizli (in Turkish).

[40] Yücel, E. (2005): Ağaçlar ve Çalılar. - Eskişehir (in Turkish).

[41] Zhang, Z., Gao, J., Kong, D., Wang, A., Tang, S., Li, Y., Pang, X. (2015): Assessing genetic diversity in Ziziphus jujuba 'Jinsixiaozao' using morphological and microsatellite (SSR) markers. - Biochemical Systematics and Ecology 61: 196-202. https://doi.org/10.1016/j.bse.2015.06.021.

[42] Zheng, Y., Wang, S. Y., Wang, C. Y., Zheng, W. (2007): Changes in strawberry phenolics, anthocyanins, and antioxidant capacity in response to high oxygen treatments. - LWT-Food Science and Technology 40(1): 49-57. 